\title{
Positional capability of a hexapod robot for machining applications
}

\author{
J. D. Barnfather ${ }^{1}$ • M. J. Goodfellow ${ }^{2}$. T. Abram ${ }^{1}$
}

Received: 19 February 2016 / Accepted: 15 June 2016 / Published online: 18 July 2016

(C) The Author(s) 2016. This article is published with open access at Springerlink.com

\begin{abstract}
In large volume manufacturing, moving heavy components around manufacturing facilities to machine features on them can represent a significant proportion of the parts final cost because the tools used for this require a high initial investment and operating costs. This motivates interest in robotic machining so that the "process-to-part" concept can be employed. However, typical industrial robots lack the positional capability of conventional equipment, which ultimately results in dimensional errors in the features machined. This research investigates accumulation of error originating from non-cutting stages of robotic machining programs, using a hexapod robot. This is done using a procedure adapted from ISO 9283-Manipulating Industrial Robots-Performance Criteria and Related Test Methods to determine positional accuracy and repeatability, i.e. systematic and random errors. This concludes that, although the robot encounters high levels of error prior to cutting, a portion of these may be offset with in-situ condition monitoring to facilitate higher tolerance machining. Potential is therefore found for using robot machining for manufacturing cost reduction in the large-scale manufacturing industries.
\end{abstract}

Keywords Robot $\cdot$ Machining $\cdot$ Hexapod $\cdot$ Error

J. D. Barnfather

joshbarnfather@hotmail.com

1 The Department of Mechanical, Aerospace and Civil Engineering, The University of Manchester, Pariser Building, Sackville Street, Manchester M13 9PL, UK

2 Rolls-Royce Plc. Civil Nuclear, PO Box 2000, Raynesway, Derby DE23 7XX, UK

\section{Introduction}

In large volume manufacturing, challenges are faced in feature machining due to the size and weight of components that the features belong to. This is costly due to the capital expenditure requirements of large machine tools and the need for heavy lifting around production facilities. An alternative approach being investigated to decrease these costs is robotic feature machining with low-cost industrial hexapod robots so that the "process-to-part" concept can be employed.

The hexapod robot format is of interest for machining applications because of stiffness benefits and noncumulative error stack up in independent links and joints, which provides improved chatter resistance and accuracy compared to serial-arm alternatives [1-6]. A limitation to robotic machining using hexapod industrial robots is that they are less able to achieve the higher machining tolerances of conventional machine tools due to relative differences in structural rigidity and tool deflection and challenges associated with control algorithms, encoder capability and component misalignments [7-14].

A widely researched aspect of hexapod-based robotic machining is kinematic modelling, which aims to accurately relate the programmed end effector positions to joint rotations and actuator extensions for program execution. Robot kinematics are discussed by Weill et al., where dimensional errors in links and joints are noted to cause assembly misalignments and therefore differences between the nominal kinematic models, used in the controller, and the real world [15]. However, kinematic modelling challenges are faced for parallel robot structures, such as hexapods, as they have a complex configuration of joints.

For example, in the work of Karimi and Nategh interpolation errors occurring as a result of non-linear mapping 
between the actuator and machining coordinate spaces in hexapod robotic machine tools are modelled and the effect of various parameters on toolpath accuracy are studied and experimentally verified. This work finds that toolpath length and direction influences the kinematic error levels [16]. Von Daake et al. propose an approach for hexapod kinematic calibration based on significantly fewer pose measurements than is typical in other work [17]. Agheli and Nategh also discuss hexapod kinematic calibration and find that optimum results are achieved when models are based on maximum observable robot error measurements, which are at the limits of the working envelope in this case [4]. This potentially introduces difficulties in identifying the highest error levels because Halaj and Kurekova conclude that positional errors vary over the working envelope [18].

The kinematics issues reported ultimately result in positional error in robot manipulation. This is of concern because Tunc et al. [19-21] show that dynamic stiffness, and potentially machining error, varies over the robots working envelope, which means that positional error between features may be a significant error contributor depending on the varying dynamic influences. However, there is a distinct lack of published studies available that characterise the overall positional error of hexapod-format industrial robotics that originate from the widely researched kinematic challenges. A contribution of this nature is desirable as this is a key indicator for process selection and benchmarking technology advancements.

This paper therefore presents the findings of experiments conducted to quantify the positional accuracy and repeatability of a Fanuc F200iB hexapod, thereby building on the referenced literature. The study aims to better understand the contributions of systematic and random errors to the overall dimensional error accumulating in machined features from non-cutting moves in machining programs. This gives an indication of capability to machine to specific tolerances by defining the robots inherent static errors.

An account of work done is presented beginning with definitions of terms and the analysis procedure used in Section 2. An overview of the equipment used, details of the experimental setup and methodology is then given in Sections 3 and 4. Finally, data is analysed and results are discussed in Section 5 and findings are summarised in Section 6.

\section{Definitions and theory}

Accuracy and repeatability is analysed according to ISO 9283 [22]. Statistically, the theory is similar to what is used in gage repeatability and reproducibility studies, which assess variance of measurement results between operators and workpieces. Whilst this may be adaptable to robot performance analysis, it is more applicable to assessing whole measurement procedures used in production rather than assessing the equipment [23].

Pose accuracy, $A P_{P}$, is defined as the difference between measured and commanded coordinates, $x_{c}, y_{c}$ and $z_{c}$, and quantifies systematic errors. These are constant and can be calibrated against when understood. Accuracy is calculated using Eq. 1, where $\bar{x}, \bar{y}$ and $\bar{z}$ are the mean coordinates of the measured poses, i.e. the barycentres. Systematic errors can occur due to axis location and orientation errors, causing offsets between theoretical and real zeros positions of encoders and positional deviations varying linearly over the workspace [24].

$A P_{P}=\sqrt{\left(\bar{x}-x_{c}\right)^{2}+\left(\bar{y}-y_{c}\right)^{2}+\left(\bar{z}-z_{c}\right)^{2}}$

Pose repeatability, $R P_{l}$, is defined as the variance between poses and accounts for random errors, which are not controllable through calibration because influencing factors are not constant. $R P_{l}$ is expressed as the radius of a sphere with the barycentre as the centre point, given by Eqs. 2-5. Here, $\bar{l}$ is the mean radius, i.e. the distance between the barycentre and measured coordinates, $j . l_{j}$ is this distance for the $j$ th measured coordinate and $S_{l}$ is the standard deviation of radii giving the spread about the mean value. $S_{l}$ predicts $3 \sigma$ of measurements are within the value computed under the same conditions.

$R P_{l}=\bar{l}+3 S_{l}$

$\bar{l}=1 / n \sum_{j=1}^{n} l_{j}$

$l_{j}=\sqrt{\left(x_{j}-\bar{x}\right)^{2}+\left(y_{j}-\bar{y}\right)^{2}+\left(z_{j}-\bar{z}\right)^{2}}$

$S_{l}=\sqrt{\frac{\sum_{j=1}^{n}\left(l_{j}-\bar{l}\right)}{n-1}}$

To expand on the analysis prescribed in ISO 9283, correlation analysis and significance testing is used to quantify the strength of visually identified linear relationships. Correlation coefficients, $R$, are computed according to the Pearson correlation analysis method [25]. $R$ is a value between -1 and +1 , indicating the degree to which a linear relationship is directly or inversely proportional. $R$ is computed as follows, where $X$ and $Y$ are the two variables under consideration, $\bar{X}$ and $\bar{Y}$ are their means, $n$ is the sample size and $i$ is the individual data point.

$$
R=\frac{\sum_{i=1}^{n}\left(X_{i}-\bar{X}\right)\left(Y_{i}-\bar{Y}\right)}{\sqrt{\sum_{i=1}^{n}\left(X_{i}-\bar{X}\right)^{2}} \sqrt{\sum_{i=1}^{n}\left(Y_{i}-\bar{Y}\right)^{2}}}
$$


To test the statistical significance of $R$ and therefore the null hypothesis that there is no correlation, $P$ values are also computed in the MATLAB function used for correlation analysis [26]. $P$ values $\leq 0.05$ indicate that $R$ has a low probability of being incorrect and that the correlation is statistically significant. A precursor to significance testing is normally distributed data.

Normality is indicated graphically using histograms with fitted ideal normal distribution curves. Normality tests are also done when sample size is $\geq 30$, as recommended by Razali et al. [27]. The Anderson-Darling normality test is chosen for this due to convenient implementation in MATLAB [26] and because it achieved a consistently high statistical power in the Razali et al. study in comparison to alternatives. Normality tests reject the null hypothesis that the data is normally distributed if the estimated probability, $P$ value, of computing the test statistic, $A^{2}$, for a normal distribution is $\leq 0.05$. The alternative hypothesis that the data is not normally distributed is rejected when the $P$ value is $\geq 0.05$. The $P$ value is determined from critical values [28, 29] of $A^{2}[26] . A^{2}$ is computed as follows, where $n$ is sample size, $i$ is the individual data point index, $F$ is the distribution function and $X$ is the dataset.

$A^{2}=-n-\sum_{i=1}^{n} \frac{2 i-1}{n}\left[\ln \left(F\left(X_{i}\right)\right)+\ln \left(1-F\left(X_{n+1-i}\right)\right)\right]$

Non-normally distributed data may occur due to outliers, process variables shifts, biases, process limits or because it has a different distribution. If this is the case, then data is transformed using the Box-Cox method [30]. Each individual value from the dataset, $y_{i}^{(\lambda)}$, is transformed by raising it to the power of $\lambda$, which is found by searching for the optimum value between -5 and 5 to achieve normality according to $A^{2}$ excluding 0 , where the natural logarithm of the dataset is taken.

$y_{i}^{(\lambda)}=\left\{\begin{array}{cc}\frac{y_{i}^{\lambda}-1}{\lambda} ; & \lambda \neq 0 \\ \log y_{i} ; & \lambda=0\end{array}\right.$

\section{Equipment and setup}

The hardware used to investigate robot positional capability was a parallel kinematic design Fanuc F200iB hexapod with stiffness benefits over serial arm alternatives [12]. This robot has six independent actuators for legs, which allows a platform to be manipulated in six degrees of freedom based on their programmed extension. Fixed to the platform is a machining spindle, which is measured using a Leica AT401 laser tracker. This instrument is calibrated to national standards and has maximum errors of $36 \mu \mathrm{m}$ specified according to ASME B89.4.19-2006, although typically observed errors can reach $7 \mu \mathrm{m}$ [31]. Robot measurements are made by mounting the laser tracker's spherically mounted retro-reflector (SMR) in the spindle as the robot tool centre point (TCP) (Fig. 1).

The laser tracker and robot coordinate systems are aligned by applying a measured rigid transformation to the trackers working coordinate frame. This puts laser tracker measurements in the same coordinate system at the robot, allowing errors between commanded and achieved robot positions to be determined up to the error and uncertainty of the laser tracker. To do this, the following steps are completed:

\section{Translation:}

1. TCP coordinates are set as the mounted retroreflectors centre point.

2. The tracker's working frame axis directions are selected to approximately match the robot's.

3. The robot is measured at its origin to acquire a vector for laser tracker origin translation (Fig. 2).

\section{Rotation:}

1. The robot is moved to points in the $\mathrm{XY}, \mathrm{YZ}$ and $\mathrm{ZX}$ planes where measurements are made.

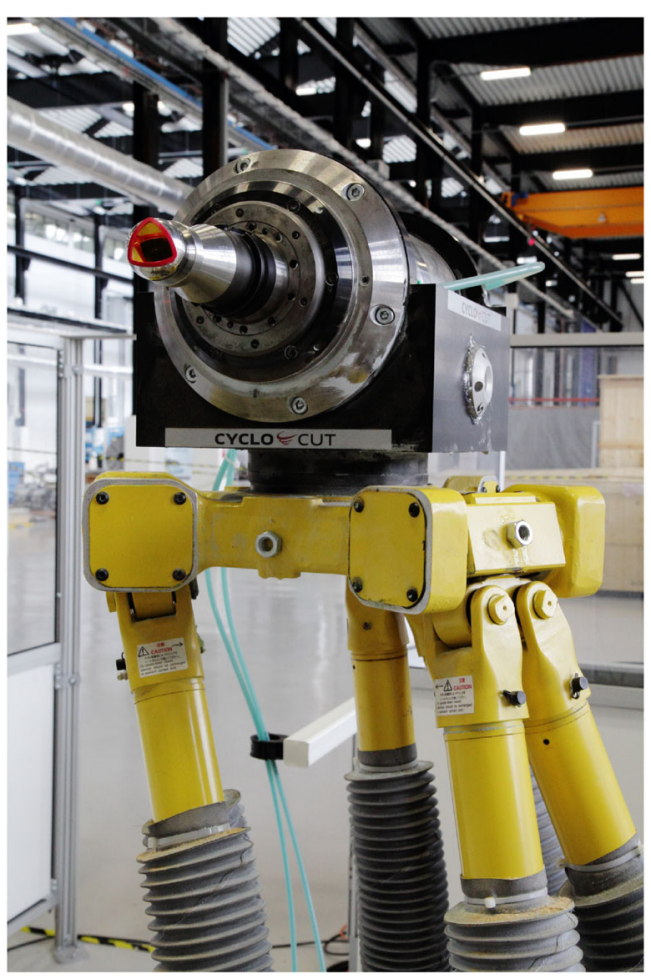

Fig. 1 Fanuc F200iB Hexapod and Gamifior Spindle 
1. Robot TCP with SMR at origin

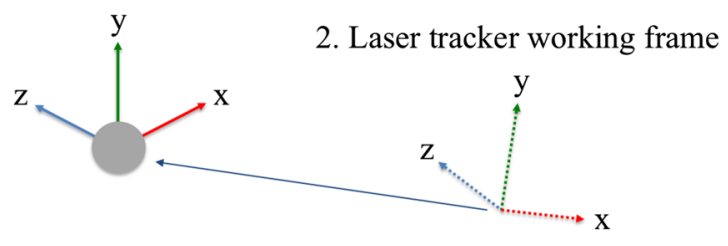

3. Translated frames

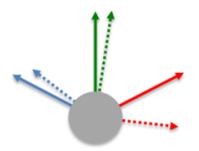

Fig. 2 Laser tracker to robot translation

2. A coordinate frame is fitted at the intersection of planes formed by these points (Fig. 3).

3. The frame is used to fine-tune the laser tracker frame orientation to match the robot, using $R_{x}, R_{y}$ and $R_{z}$ (Fig. 4).

\section{Methodology}

A methodology adapted from ISO 9283-Manipulating Industrial Robots-Performance Criteria and Related Test Methods [22, 32] is followed initially and then extended to explore performance further and validate conclusions. In the first stage, adaptations were made to the test geometry specified as the robot is moved to 9 poses, $P 1-9$, rather

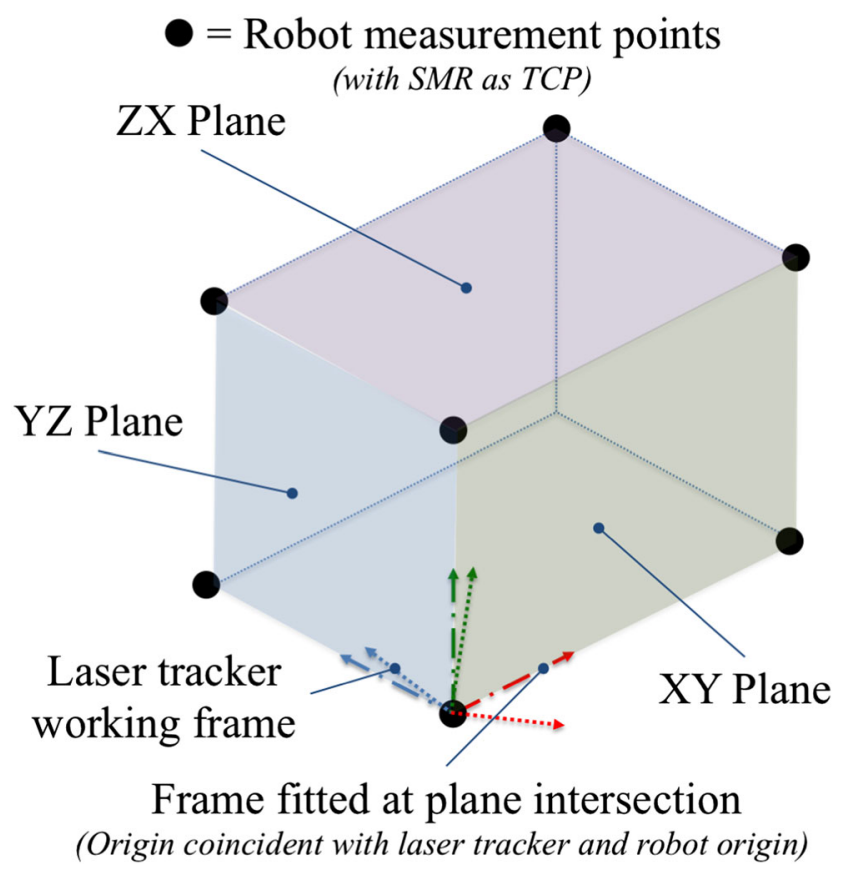

Fig. 3 Laser tracker to robot rotation measurement



Fig. 4 Laser tracker to robot rotation values

than 4 to assess a larger portion of the working envelope. Poses are arranged in the largest available cuboid in the centre of the working volume, $500 \times 250 \times 185 \mathrm{~mm}$, as shown below, although the absolute limits extend beyond this [33] (Fig. 5). Inline with the standard, the poses have been offset by $10 \%$ of the diagonal distance. However, whilst it does give an efficient insight into performance, the geometry is not necessarily optimised and should ideally be designed to expose equipment specific errors. In this case, the cutting tool orientation does not maintain perpendicularity with the diagonal planes as specified in the standard as 3-axis machining is of interest .

To conduct the experiment, the program was run for 30 cycles at 20, 100 and $200 \mathrm{~mm} / \mathrm{s}$ with measurements taken at each pose in a workshop floor environment lasting a up to $109 \mathrm{~min}$. Feed rate is considered as it is suggested to be

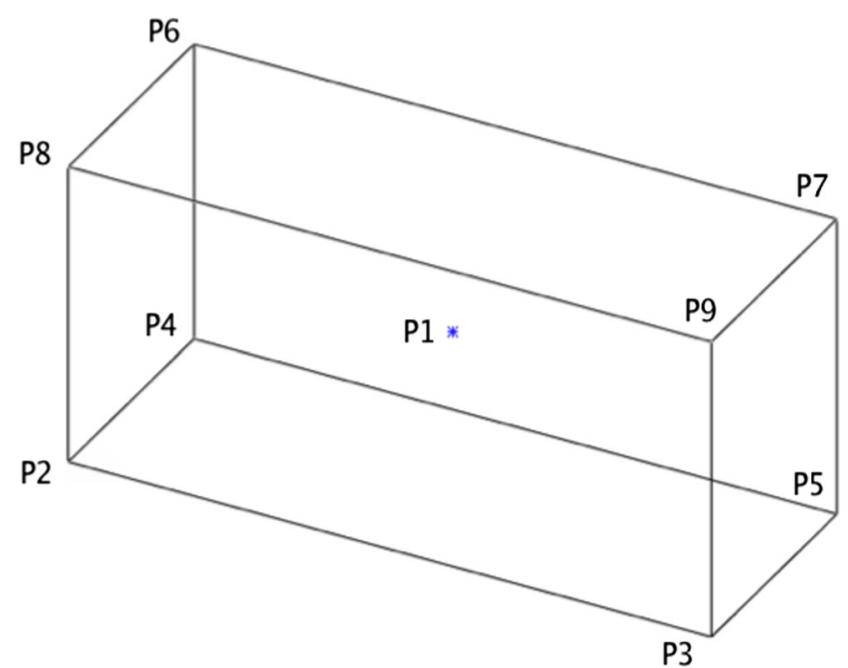

Fig. 5 Programmed poses 
a potential influence on error by the standard and values are initially selected to cover those likely for use in non-cutting moves of machining programs. Another adaptation to the standard is that the robot is not $100 \%$ loaded as this is not representative of machining conditions.

To expand on the standard procedure, the effect of feed rate, performance drift and robot configuration on error is also investigated, which represent the user selected static variables of a robot machining program. The maximum feed rate of the robot is $300 \mathrm{~mm} / \mathrm{s}$ in the vertical direction and $1500 \mathrm{~mm} / \mathrm{s}$ in the horizontal direction [33]. Additional experimentation is done by measuring the robot moving to 2 poses 30 times each at 15 feed rates covering the full range. Considering only two poses minimises the error occurring from robot configuration and potentially enables feed rates to be optimised in between cutting stages of machining programs to minimise error.

Performance drift over time is studied further using a similar procedure as the robot is moved to two poses $>3000$ times, over $\sim 5.5 \mathrm{~h}$. This may expose thermally induced performance drift, which can cause significant errors due to component expansion and contraction due to fluctuating heat sources originating from the environment, the part, robot axes, spindle and the cutting process $[34,35]$. For example, spindle expansion could exceed $100 \mu \mathrm{m}$ from cold start conditions [36]. Nevertheless, the investigation done is preliminary only and should be expanded by conducting experiments to analyse the specific temperature profiles of individual components in the robotic machine tool if an error trend with time is observed.

To further study configuration sensitivity and errors throughout the working volume, systematic errors are assessed by calculating the difference between commanded and measured poses arranged in grids with $10-\mathrm{mm}$ spacing between each in each axis, i.e. error maps. Robot configuration is thought to have an impact on error because of the kinematic modelling challenges highlighted in the Section 1.

\section{Results and analysis}

Mean systematic positional error, i.e. accuracy, from the first stage of experiments is shown in Fig. 6, plotted against feed rate at each point. Error bars represent three standard deviations of the measurements to show random error, i.e. repeatability, which also encompasses laser tracker uncertainty. Accuracy ranged from 16.1 to $417.9 \mu \mathrm{m}$ with relatively low variation, suggesting that robot configuration contributes to systematic error due to the kinematic issues referenced in the introduction. This potentially indicates that the kinematic model used for control does not accurately represent the true robot dimensions or encoder readings.

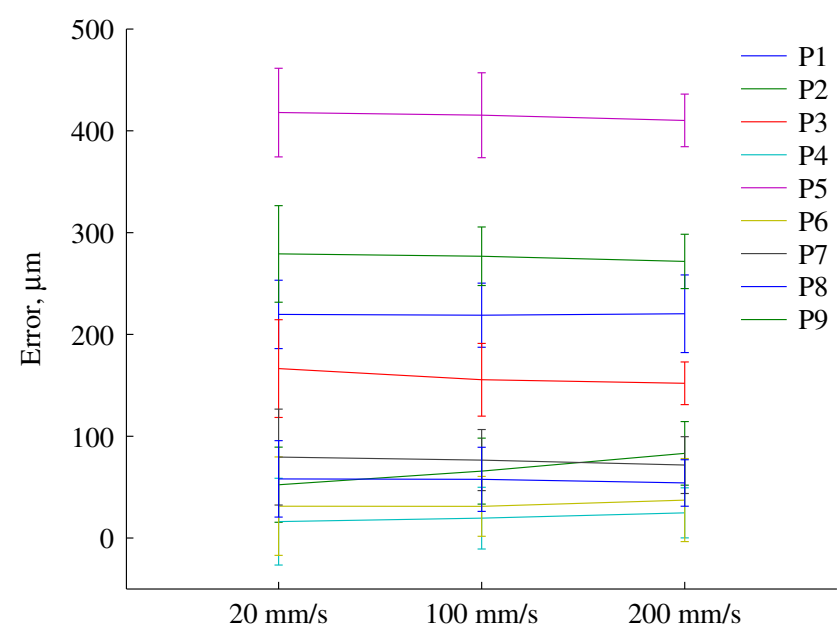

Fig. 6 Positional error

Random errors are consistently within the manufacturers specification of $100 \mu \mathrm{m}$ [33]. Basic results are expanded on to study error accumulation in robotic machining. These are discussed in the context of feed rate, performance drift and robot configuration in Sections 5.1-5.3.

\subsection{Effect of feed rate}

Initial experiments show systematic error to be consistent over the feed rates tested, but that random error is more sensitive. Tracker uncertainty means that the effect of feed rate on systematic error cannot be assessed as the difference is so low. Further insight is gained with extended studies, as shown in Fig. 7 where the sum of overall errors for two positions are plotted against feed rate.

Feed rate is now observed to be associated with a distinct error level at $100 \mathrm{~mm} / \mathrm{s}$, although variance at other feed rates

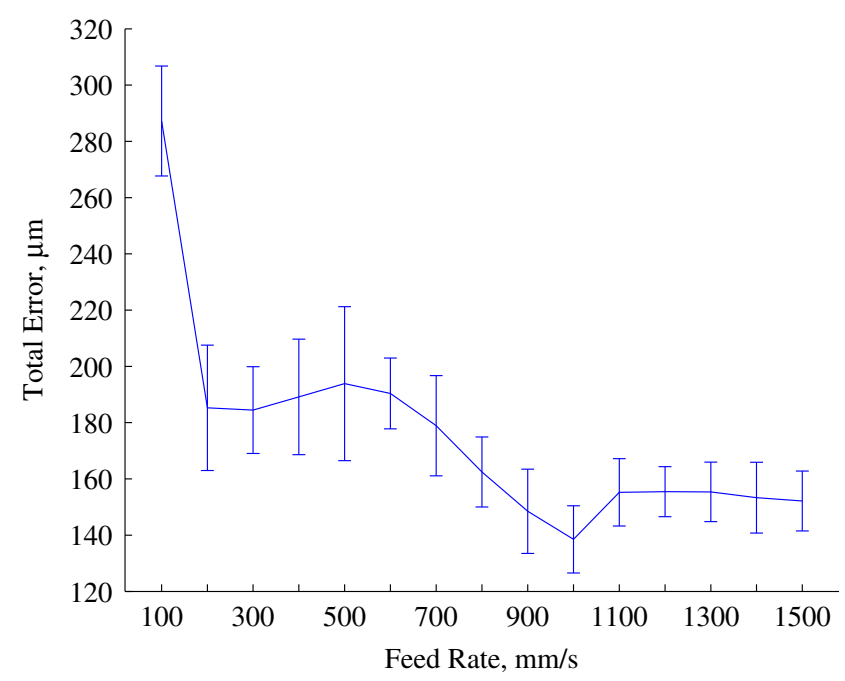

Fig. 7 Feed rate effect on error 



Fig. 8 Systematic error over experiment progress

back up initial results and suggest a relationship with error does not exist to a degree that could be exploited for error reduction during parameter selection for non-cutting moves in machining programs. This is highlighted by the error bars, which represent the random errors associated with the mean systematic errors at each feed rate.

\subsection{Performance drift}

No evidence of performance drift is seen over the time scale of initial experiments using the methodology most closely based on ISO 9283. Drift may of been observed if robot components were fluctuating in temperature enough during operation and experiencing thermally induced dimensional change to a degree that would impact the position of the tool centre point. This is shown in Fig. 8, where individual measurements for each pose are broken down into the magnitudes of $x y z_{j}$, with the $x y z_{c}$ subtracted from them and plotted against measurement number to show the contributions to overall accuracy.

Error variation over time from extended performance drift experiments is shown in Fig. 9, where the sum of systematic and random errors at each of the two poses tested are plotted over time. Overall, error fluctuation within a range of $\sim 70 \mu \mathrm{m}$ for both points is observed, which may partially reflect laser tracker uncertainty and make robot performance drift of minimal concern. This would make robot performance drift of minimal concern. Whilst laser tracker thermal, vibration or humidity induced drift may be a concern [37], this cannot be confirmed without running experiments that study measurement variations over time alongside these potential contributors, independently of the robotics application.

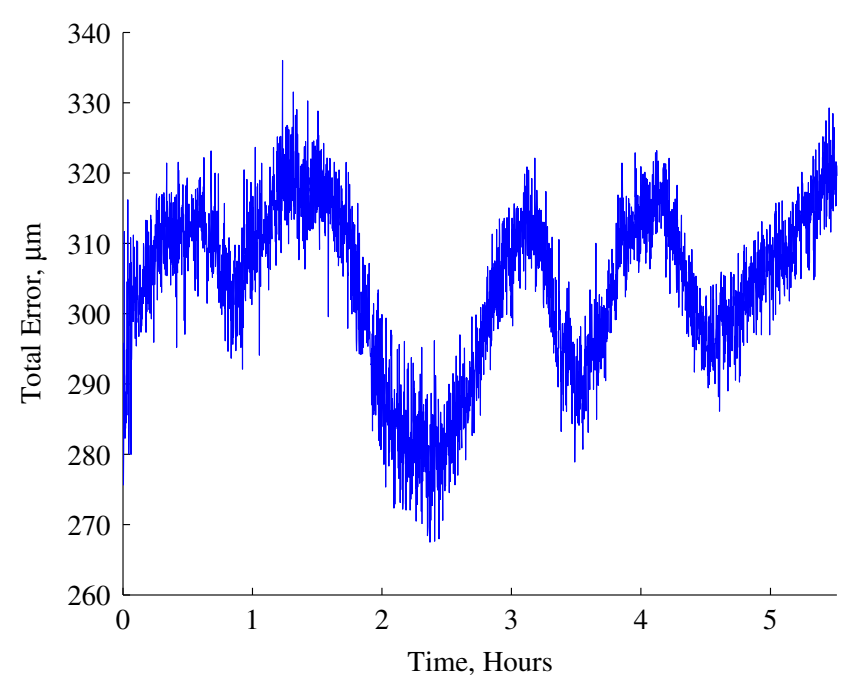

Fig. 9 Positional error drift

If error fluctuations do reflect robot behaviour, the relationship is non-linear with time and highlights the complexity of calibration. Calibration would rely on significant modelling effort and in-situ condition monitoring, as used in $[38,39]$, to achieve an adaptive kinematic model reactive to all dynamics of robotic machining. Challenges associated with this are discussed in [40-44] in the context of thermal error drift. Nevertheless, Pan et al. [45] suggests that robotic machining error arising from structural rigidity is of higher priority if one particular challenge was to be focused on.

\subsection{Configuration sensitivity}

Initial experimental results, given in Fig. 6, highlight systematic error sensitivity to robot configuration but that random errors are independent of robot configuration. In the remaining tables and figures, the results of the error mapping exercise done to expand the investigation are summarised. Error maps are expressed in the cartesian coordinate used in programming, i.e. the robot coordinate system, rather than in terms of individual actuators extensions and joint rotations. This gives insight into how potentially complex interactions of error sources ultimately affect suitability for specific machining applications, which is not intuitive if errors were mapped in relation to actuators and joint motion. Firstly, it is observed that the majority of errors are dispersed over a range that is unacceptable in high tolerance applications, estimating that $95 \%$ of errors in will fall between 56.62 and $588.74 \mu \mathrm{m}$, as shown in Table 1 .

The influence of individual axes on systematic error is seen in colour maps, in Figs. 10 and 11, showing the outermost robot positions on the cuboid volume and its central cross sections, respectively. These indicate that systematic error is not correlated with $\mathrm{Y}$ and $\mathrm{Z}$ motion but that there is 
Table 1 Error map summary statistics

\begin{tabular}{llll}
\hline Mean & $322.68 \mu \mathrm{m}$ & Total points & 5589 \\
Median & $312.33 \mu \mathrm{m}$ & $A_{i}^{2}$ & 54.805 \\
Min & $14.50 \mu \mathrm{m}$ & $P_{i}$ & 0.0005 \\
Max & $816.06 \mu \mathrm{m}$ & $A^{2}$, & 40.378 \\
Std. Dev & $133.00 \mu \mathrm{m}$ & $P^{,}$ & 0.0005 \\
\hline
\end{tabular}

a linearly increasing error exceeding values gained in initial experiments in the $\mathrm{X}$ axis. Errors against the overall trend are outliers and there are gaps where no measurement has been obtained. Other outliers occur due to mis-timed measurements before or after the robots stable position, i.e. no movement beyond a $20 \mu \mathrm{m}$ radius for $1.2 \mathrm{~s}$. These are identified by double points close to the commanded coordinate undistinguishable as more or less correct so only the first is used for error calculation. The data gained is still useful because the anomalies represent only $3 \%$ of the dataset and occur randomly.

To quantify apparent relationships, normality testing of the error map dataset is done to prepare for correlation analysis. Initial Anderson-Darling tests, $A_{i}^{2}$, indicate nonnormality, justifying transformation to fulfil assumptions for this. Transformation achieves an improvement, $A^{2}$, but tests reject the null hypothesis with $P$ values, $P_{i}$ and $P$, being $<0.05$ for both initial and transformed data, respectively. This is also given in Table 1.

In this case, approximate normality is acceptable according to the central limit theorem which states that, regardless

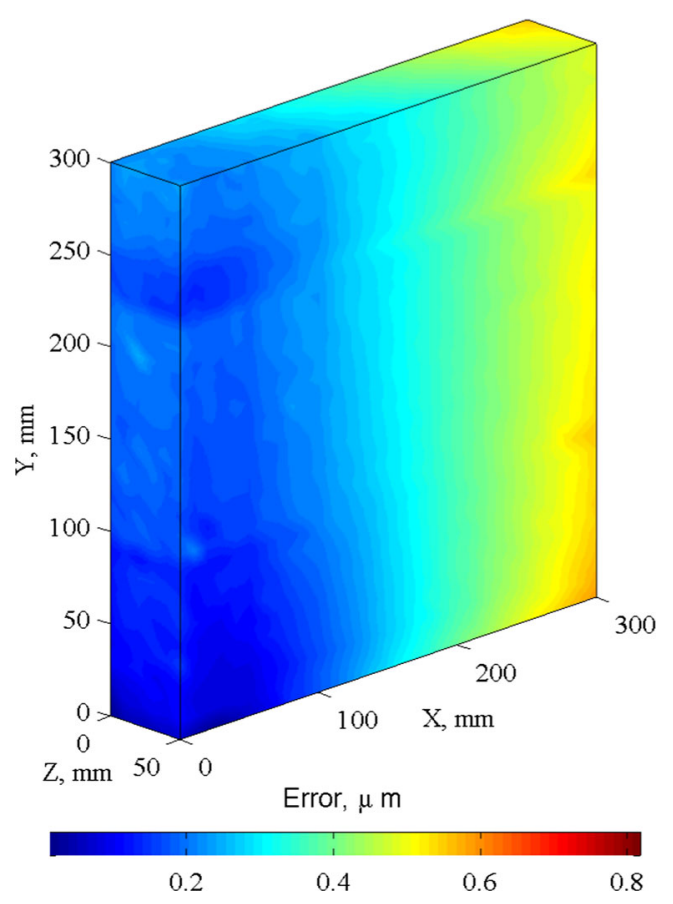

Fig. 10 Error map block

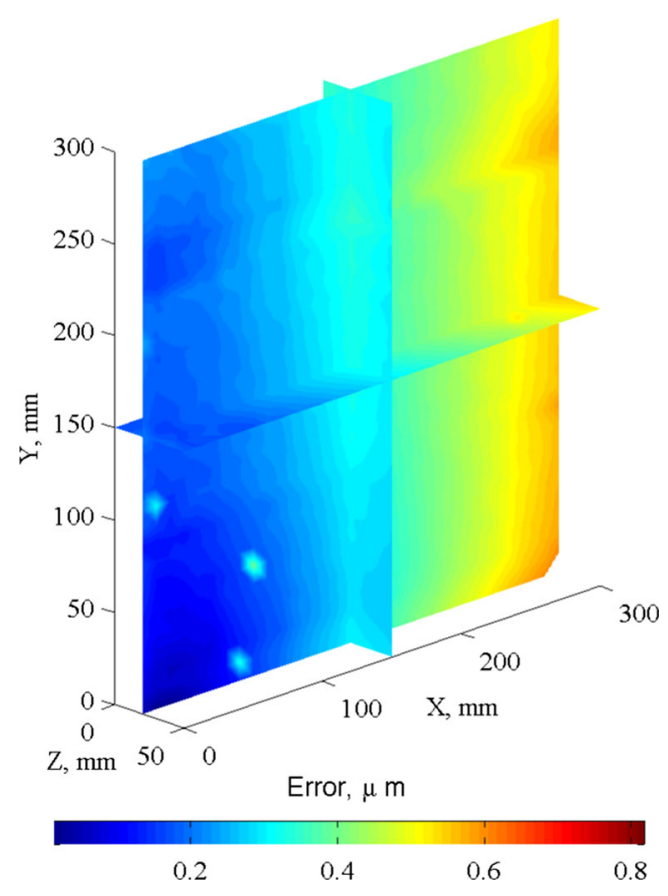

Fig. 11 Error map cross sections

of the shape of the population distribution, if the sample size is large then the sampling distribution is normal [25]. This is proved in Fig. 12 where the frequency of the means of 1000 random samples of the population of errors are plotted, sized at 100 data points each. An Anderson-Darling test validates the normality assumption for significance testing as part of correlation analysis, computing $A^{2}$ at 0.52 with a $P$ value of 0.19 .

Correlation between axis and error is subsequently validated by computing correlation coefficients and corresponding significance levels. These are shown below in Table 2 and validate that, whilst there is a relatively small impact

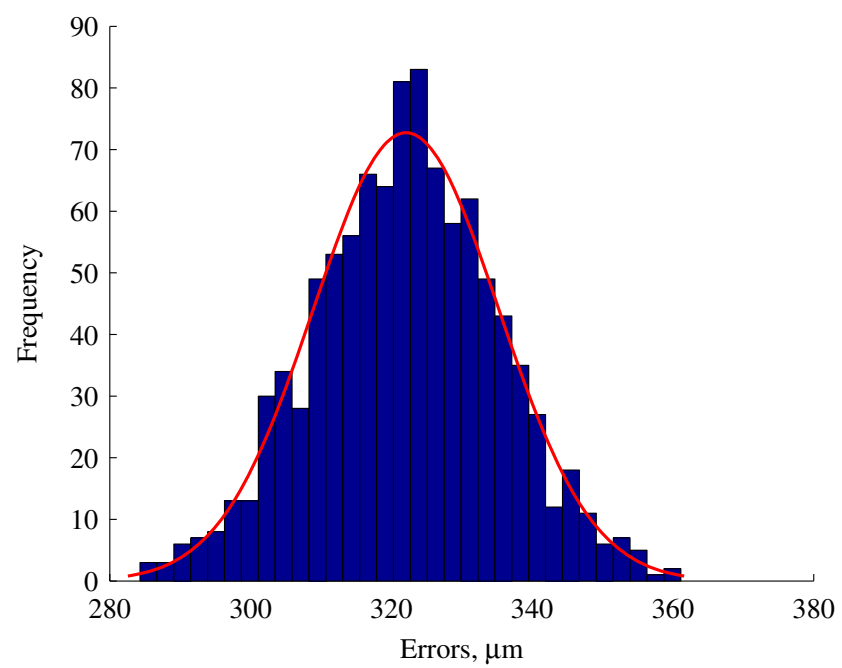

Fig. 12 Error map sampling distribution 
Table 2 Axis correlation to error

\begin{tabular}{lllll}
\hline Axis & $R$ & $\begin{array}{l}\text { Lower } \\
\text { limit }\end{array}$ & $\begin{array}{l}\text { Upper } \\
\text { limit }\end{array}$ & $P$ \\
\hline $\mathrm{X}$ & 0.9636 & 0.9617 & 0.9655 & 0.0000 \\
$\mathrm{Y}$ & 0.1134 & 0.0875 & 0.1392 & $1.8 \mathrm{e}-17$ \\
$\mathrm{Z}$ & -0.0874 & -0.1133 & -0.0613 & $5.9 \mathrm{e}-11$ \\
\hline
\end{tabular}

on systematic error as the robot is moved along its $\mathrm{Y}$ and $\mathrm{Z}$ axes, this is most prominent in the $\mathrm{X}$ axis. This observation supports [4], where the edge of the working envelope is noted to be a region of high error, as the extreme $+\mathrm{X}$ position corresponds to the maximum extension of each individual robot actuator in the experiment. Results are shown to have a low probability of incorrectly concluding correlation with each $P$ value approaching 0 . Because the relationship shown is linear, this problem could be solved with a simple calibration. Nevertheless, it should be considered that this reflects error in the whole process, i.e. the contributions from the robot, the laser tracker and the alignment process. Validation by running the experiment in the reverse order, i.e. motion from $-\mathrm{X}$ to $+\mathrm{X}$, may therefore prove to be insightful.

\section{Summary and conclusions}

Robot positional experiments have been conducted to quantify the systematic and random errors of non-cutting moves in robotic machining programs. Basic and extended methodologies based on an adaptation of the ISO 9283 procedure were used to assess a Fanuc F200iB robot. Overall positional errors are frequently observed to reach several hundred microns. Feed rate is not found to have a significant impact on error but performance drift studies over time are inconclusive, with a minimal impact if any. The main impact on positional error in the process is found to be robot configuration.

The robot tested cannot currently machine to high tolerance due to the static errors encountered before cutting even begins. However, the main conclusion is that systematic error is more dominant than random error, which implies that total error can be reduced. Whilst the systematic errors can be calibrated against, a challenge faced is that all static and dynamic process variables need to be understood and modelled to do this, which is highly complex. This necessitates the use of in situ process monitoring to counteract the sum systematic errors rather than individual contributors.

To expand this investigation, it is recommended that machining dynamics are accounted for by conducting machining trials [46]. This would allow real-world performance to be judged for specific machining applications.
Acknowledgments The author of this paper would like to acknowledge Rolls-Royce Civil Nuclear and the Engineering and Physical Sciences Research Council for the provision of funding and to the Nuclear AMRC for access to equipment and technical support. The views expressed in this paper are those of the authors and not necessarily those of the funding bodies or other organisations mentioned.

Open Access This article is distributed under the terms of the Creative Commons Attribution 4.0 International License (http:// creativecommons.org/licenses/by/4.0/), which permits unrestricted use, distribution, and reproduction in any medium, provided you give appropriate credit to the original author(s) and the source, provide a link to the Creative Commons license, and indicate if changes were made.

\section{References}

1. Fassi I, Wiens G Multiaxis machining PKMs and traditional machining centers. J Manuf Process. doi:10.1016/ S1526-6125(00)70008-9

2. Bi Z, Jin Y (2011) Kinematic modeling of Exechon parallel kinematic machine. Robot Comput Integr Manuf 27(1):186-193. doi:10.1016/j.rcim.2010.07.006

3. Bi Z, Wang L (2009) Optimal design of reconfigurable parallel machining systems. Robot Comput Integr Manuf 25(6):951-961. doi:10.1016/j.rcim.2009.04.004

4. Agheli M, Nategh M (2009) Identifying The kinematic parameters of hexapod machine tool. World Acad Sci Eng Technol 3(52):380 385

5. Chanal H, Duc E, Hascoët JY, Ray P (2009) Reduction of a parallel kinematics machine tool inverse kinematics model with regard to machining behaviour. Mech Mach Theory 44(7):1371-1385. doi:10.1016/j.mechmachtheory.2008.11.004

6. Kanaan D, Wenger P, Chablat D (2009) Kinematic analysis of a serial-parallel machine tool The VERNE machine. Mech Mach Theory 44(2):487-498. doi:10.1016/ j.mechmachtheory.2008.03.002. arXiv:hep-th/0811.4733

7. Olabi A, Béarée R, Gibaru O, Damak M (2010) Feedrate planning for machining with industrial six-axis robots. Control Eng Pract 18(5):471-482. doi:10.1016/j.conengprac.2010.01.004

8. Zargarbashi S, Khan W, Angeles J (2012) The Jacobian condition number as a dexterity index in 6R machining robots. Robot Comput Integr Manuf 28(6):694-699. doi:10.1016/j.rcim.2012.04.004

9. Nubiola A, Bonev IA (2013) Absolute calibration of an ABB IRB 1600 robot using a laser tracker. Robot Comput Integr Manuf 29(1):236-245. doi:10.1016/j.rcim.2012.06.004

10. Zargarbashi S, Khan W, Angeles J (2012) Posture optimization in robot-assisted machining operations. Mech Mach Theory 51:7486. doi:10.1016/j.mechmachtheory.2011.11.017

11. Abele E, Weigold M, Rothenbücher S (2007) Modeling and identification of an industrial robot for machining applications. CIRP Ann Manuf Technol 56(1):387-390. doi:10.1016/j.cirp.2007.05.090

12. Pessi $\mathrm{P}, \mathrm{Wu} \mathrm{H}$, Handroos $\mathrm{H}$, Jones L (2007) A mobile robot with parallel kinematics to meet the requirements for assembling and machining the ITER vacuum vessel. Fusion Eng Des 82(15-24):2047-2054. doi:10.1016/j.fusengdes.2007.06.012

13. Pan Z, Zhang H, Zhu Z, Wang J (2006) Chatter analysis of robotic machining process. J Mater Process Technol 173(3):301-309. doi:10.1016/j.jmatprotec.2005.11.033 
14. Dumas C, Caro S, Garnier S, Furet B (2011) Joint stiffness identification of six-revolute industrial serial robots. Robot Comput Integr Manuf 27(4):881-888. doi:10.1016/j.rcim.2011.02.003

15. Weill R, Shani B (1991) Assessment of accuracy in relation with geometrical tolerances in robot links. CIRP Ann Manuf Technol 40(1):395-399. doi:10.1016/S0007-8506(07)62015-0

16. Karimi D, Nategh MJ (2014) Kinematic nonlinearity analysis in hexapod machine tools Symmetry and regional accuracy of workspace. Mech Mach Theory 71:115-125. doi:10.1016/j.mechmachtheory.2013.09.007

17. Von Daake A, Vetter C, Böhm E, Zirn O (2013) Contribution to calibration of hexapod positioning units in industrial environment. Precis Eng 37(1):73-80. doi:10.1016/j.precisioneng.2012.07.002

18. Halaj M, Kureková E (2009) Positioning accuracy of nonconventional production machines-an introduction. In: Proceedings of XIX IMEKO World Congress, Lisbon, pp 2099-2102

19. Tunc LT, Barnfather J (2014) Effects of hexapod robot dynamics in milling. In: 11th international conference on high speed machining, MM science journal, Prague

20. Tunc LT, Shaw J (2015) Experimental study on investigation of dynamics of hexapod robot for mobile machining. Int $\mathbf{J}$ Adv Manuf Technol:817-830. doi:10.1007/s00170-015-7600-6

21. Tunc LT, Shaw J Investigation of the effects of Stewart platformtype industrial robot on stability of robotic milling. Int $\mathbf{J}$ Adv Manuf Technol. doi:10.1007/s00170-016-8420-z

22. BS EN ISO 9283:1998 Manipulating Industrial Robots - Performance Criteria and Related Test Methods (1998)

23. Peruchi RS, Balestrassi PP, de Paiva AP, Ferreira JR, de Santana Carmelossi M (2013) A new multivariate gage R\&R method for correlated characteristics. Int J Prod Econ 144(1):301-315. doi:10.1016/j.ijpe.2013.02.018

24. Alami Mchichi N, Mayer JRR (2014) Axis location errors and error motions calibration for a five-axis machine tool using the SAMBA method. Procedia CIRP 14:305-310. doi:10.1016/j.procir.2014.03.088

25. Spiegel M, Schiller J, Srinivasan A (2001) Probability and statistics, 1st edn. Mcgraw-hill

26. MATLAB (2013) MATLAB Statistics Toolbox Users Guide R2013a

27. Razali NM, Wah YB (2011) Power comparisons of ShapiroWilk, Kolmogorov-Smirnov, Lilliefors and Anderson-Darling tests. Journal of Statistical Modeling and Analytics 2(1):21-33

28. Stephens MA (1974) EDF Statistics for goodness of fit and some comparisons. J Am Stat Assoc 69(347):730-737. doi:10.1080/01621459.1974.10480196

29. Stephens MA (1976) Asymptotic results for goodness-of-fit statistics with unknown parameters. Ann Stat 4(2):357-369. doi:10.1214/aos/1176343411

30. Sakia RM (1992) The Box-Cox transformation technique: a review. The Statistician 41(2):169. doi:10.2307/2348250

31. Leica (2010) Leica Absolute Tracker AT401 ASME B89.4.192006 Specification
32. Barnfather JD, Goodfellow MJ, Abram T (2016) A performance evaluation methodology for robotic machine tools used in large volume manufacturing. Robot Comput Integr Manuf 37:49-56. doi:10.1016/j.rcim.2015.06.002. http://linkinghub.elsevier.com/ retrieve/pii/S0736584515000708

33. Fanuc (2005) Fanuc F-200iB Hexapod Datasheet

34. Bryan J, Lawrence JB, National L, Charlton T, Detroit DEA, Raho HS, Roy ELM (1990) International status of thermal error research (1990). CIRP Ann Manuf Technol 39(2):645-656. doi:10.1016/S0007-8506(07)63001-7

35. BS ISO 230-3:2007 Test code for machine tools - Part 3: Determination of thermal effects (2007)

36. Chen JS, Hsu WY (2003) Characterizations and models for the thermal growth of a motorized high speed spindle. Int J Mach Tools Manuf 43(11):1163-1170. doi:10.1016/ S0890-6955(03)00103-2

37. Lassila A, Kari M, Koivula H, Koivula U, Kortström J, Leinonen E, Manninen J, Manssila J, Mansten T, Meriläinen T, Muttilainen J, Nissilä J, Nyblom R, Riski K, Sarilo J, Isotalo H (2011) Design and performance of an advanced metrology building for MIKES. Measurement 44(2):399-425. doi:10.1016/j.measurement.2010.10.013

38. Weck M, McKeown P, Bonse R, Herbst U (1995) Reduction and compensation of thermal errors in machine tools. CIRP Ann Manuf Technol 44(I):589-598. doi:10.1016/S0007-8506(07)60506-X

39. Zhang T, Ye W, Liang R, Lou P, Yang X (2013) Temperature variable optimization for precision machine tool thermal error compensation on optimal threshold. Chinese Journal of Mechanical Engineering 26(1):158-165. doi:10.3901/CJME.2013.01.158

40. Yun WS, Kim SK, Cho DW (1999) Thermal error analysis for a CNC lathe feed drive system. Int J Mach Tools Manuf 39(7):10871101

41. Gong C, Yuan J, Ni J (2000) Nongeometric error identification and compensation for robotic system by inverse calibration. Int $\mathrm{J}$ Mach Tools Manuf 40(14):2119-2137

42. Yang JG, Ren YQ, Liu GL, Zhao HT, Dou XL, Chen WZ, He SW (2005) Testing, variable selecting and modeling of thermal errors on an INDEX-G200 turning center. Int J Adv Manuf Technol 26(7-8):814-818

43. Oiwa T (2005) Error compensation system for joints, links and machine frame of parallel kinematics machines. Int J Robot Res 24(12):1087-1102

44. Pahk HJ, Lee SW, Kwon HD (2001) Thermal error measurement and modelling techniques for the five-degree-of-freedom spindle drifts in computer nurmerically controlled machine tools. Proc Inst Mech Eng 215:469-485

45. Pan Z, Zhang H (2009) Improving robotic machining accuracy by real-time compensation, pp 4289-4294

46. Cheng K (2009) Machining dynamics: theory, applications and practices 